\title{
A RATIO LIMIT THEOREM FOR CONTRACTION PROJECTIONS AND APPLICATIONS
}

\author{
by P. E. KOPP \\ (Received 9 August, 1971)
}

1. The similarities between martingale convergence theory and pointwise ergodic theory are now well known $[5,7,9,10]$. In [5] the similarity between the proofs of the HopfDunford-Schwartz individual ergodic theorem and the martingale convergence theorem is systematically exploited to produce very general " maximal ergodic" inequalities for certain sequences of contractions on $L^{1}$-spaces. A different approach by Rota [10] and Rao [9] leads to a unified convergence theory for martingales and Abel limits. Bishop [1] has produced "upcrossing" inequalities which yield both the Chacon-Ornstein theorem [4] and the martingale convergence theorem.

In the present note a " ratio limit" theorem, or analogue of the Chacon-Ornstein result is proved for certain sequences of positive contractions on $L^{1}$. The proof is based upon Hopf's proof [4] of the Chacon-Ornstein theorem and is in the spirit of the analogy exploited in [5]. The set where convergence obtains is characterised in the case when the contractions are also projections. This is true when we are dealing with decreasing martingales, so that the present theorem can be regarded as a "ratio " martingale convergence theorem.

In the last section the ratio limit theorem is applied to sequences of contractive projections satisfying a uniform integrability condition, to prove an extension of the Doob martingale convergence theorem. The treatment here was suggested by a paper of Ito [6] on the individual ergodic theorem, though the method of proof here differs substantially from his methods.

I should like to thank my supervisor, Dr David Edwards, for suggesting this problem and for his valuable guidance throughout. I am also indebted to Dona Strauss and Fred Yeadon for many interesting conversations on the material in the last section.

2. A "maximal ergodic" lemma. Let $(\Omega, \mathscr{B}, \mu)$ be a complete $\sigma$-finite measure space and let $L^{1}=L^{1}(\Omega, \beta, \mu)$ denote the space of (equivalence classes of) $\mu$-integrable real-valued functions on $\Omega$. A contraction $T$ is a linear operator $T: L^{1} \rightarrow L^{1}$ with $\|T\|_{1} \leqq 1$. A sequence of contractions $\left\{T_{n}\right\}_{n \geqq 0}$ on $L^{1}$ is called decreasing if $T_{n+1} T_{n}=T_{n+1}$ for all $n \geqq 0$. By induction, this implies that $T_{m} T_{n}=T_{m}$ for all $m>n$. In particular, if $f \in L^{1}$ and $T_{n} f=E\left(f \mid \mathscr{B}_{n}\right)$, where the $\sigma$-subalgebras $\mathscr{B}_{n}$ of $\mathscr{B}$ are decreasing with $n,\left\{T_{n}\right\}$ is a decreasing sequence of contractive projections on $L^{1}$. For decreasing sequences of contractions on $L^{1}$, we prove an inequality that is analogous to the maximal ergodic theorem $[4,5]$ both in statement and proof. (Throughout, $B^{\prime}=\Omega \backslash B$, for any $B \subseteq \Omega$.)

LemMa 1. Let $\left\{T_{n}\right\}_{n \geqq 0}$ be a decreasing sequence of positive contractions on $L^{1}$, and, for $f \in L^{1}$, let $A_{f}=\left\{\omega: \limsup \left(T_{n} f\right)(\omega)>0\right\}$. Let $B \subset A_{f}$ be measurable. Then

$$
\int_{B} f d \mu+\int_{B^{\prime}} f^{+} d \mu \geqq 0 .
$$


- Proof. Define $h_{0}=f, h_{i+1}=T_{i} h_{i}^{+}-h_{i}^{-}(i=0,1,2, \ldots)$. Then $h_{i+1}^{+}-h_{i+1}^{-}=T_{i} h_{i}^{+}-h_{i}^{-}$, and so

$$
h_{i+1}^{-} \leqq h_{i}^{-} \text {, }
$$

since $T_{i} \geqq 0$ and the decomposition $g=g^{+}-g^{-}$is minimal. Also $h_{i}-h_{i+1}=h_{i}^{+}-T_{i} h_{i}^{+}=$ $\left(1-T_{i}\right) h_{i}^{+}$. Hence, by summation of the first $n$ of these equations, we obtain

$$
f-h_{n}=\sum_{i=0}^{n-1}\left(1-T_{i}\right) h_{i}^{+}
$$

But $\left\{T_{n}\right\}$ is decreasing; so

Hence

$$
T_{n} f=T_{n} h_{n}+T_{n}\left(\sum_{l=0}^{n-1}\left(1-T_{i}\right) h_{i}^{+}\right)=T_{n} h_{n} .
$$

$$
T_{n} f \leqq T_{n} h_{n}^{+}=h_{n+1}^{+}+\left(h_{n}^{-}-h_{n+1}^{-}\right) \text {. }
$$

For given $\varepsilon>0$, let $B_{\varepsilon}^{m}=\left\{\omega:\left(T_{m} f\right)(\omega)>\varepsilon\right\}$ and let $B_{\varepsilon}=\limsup B_{\varepsilon}^{m}$. Then (4) implies that, for each $\omega \in B_{s}^{m}$, we have either (a) $h_{m+1}^{+}(\omega)>0$ (and hence $\left.h_{m+1}^{-}(\omega)=0\right)$ or $(\mathrm{b})\left(h_{m}^{-}-h_{m+1}^{-}\right)(\omega)>\varepsilon$. If $\omega \in B_{\varepsilon}$, then (a) or (b) must hold for infinitely many $m$. We show that, if (b) holds infinitely often on a set $N_{\varepsilon} \subseteq B_{\varepsilon}$, then $\mu\left(N_{\varepsilon}\right)=0$. Choose $\omega$ such that $f^{-}(\omega)<\infty$. As $f^{-}(\omega)=$ $h_{0}^{-}(\omega) \geqq h_{m}^{-}(\omega) \geqq h_{m+1}^{-}(\omega) \geqq 0$, (b) can only hold finitely often for $\omega$, since otherwise there is a subsequence $\left\{m_{r}\right\}$ of natural numbers such that $f^{-}(\omega) \geqq r \varepsilon+h_{m_{r}}^{-}(\omega)$ for each $r \geqq 1$, and hence, choosing $r$ so large that $f^{-}(\omega)<r \varepsilon$, we obtain the contradiction $h_{m_{r}}^{-}(\omega)<0$. So $N_{\varepsilon} \subseteq B_{\varepsilon} \cap\left\{\omega: f^{-}(\omega)=\infty\right\}$, which is $\mu$-null. Hence there is a null set $N_{\varepsilon} \subseteq B_{\varepsilon}$ such that (a) holds for infinitely many values of $m$ at each point of $B_{e} \backslash N_{e}$. But $A_{f}=\bigcup_{n=1}^{\infty} B_{1 / n}$; so the null set $N=\bigcup_{n=1}^{\infty} N_{1 / n}$ is such that $h_{n}^{-}(\omega) \downarrow 0$ throughout $A_{f} \mid N$. Now $f+h_{n}^{-}=h_{n}^{+}+\sum_{i=0}^{n-1}\left(1-T_{i}\right) h_{i}^{+}$ by (2); so

$$
\int\left(f+h_{n}^{-}\right) d \mu=\int h_{n}^{+} d \mu+\sum_{i=0}^{n-1} \int\left(1-T_{i}\right) h_{i}^{+} d \mu \geqq \int h_{n}^{+} d \mu \geqq 0,
$$

as the $T_{i}$ are positive contractions. Now let $B \subseteq A_{f}(B \in \mathscr{B})$. Then

$$
\int_{B} f d \mu+\int_{B^{\prime}}\left(f^{+}-f^{-}\right) d \mu+\int_{B^{\prime}} h_{n}^{-} d \mu \geqq-\int_{B} h_{n}^{-} d \mu,
$$

so that $\int_{B} f d \mu+\int_{B^{\prime}} f^{+} d \mu+\int_{B^{\prime}}\left(h_{n}^{-}-h_{0}^{-}\right) d \mu \geqq-\int_{B} h_{n}^{-} d \mu$. But $h_{n}^{-}-h_{0}^{-} \leqq 0$ everywhere and $h_{n}^{-} \downarrow 0$ a.e. on $B$. So $\int_{B} f d \mu+\int_{B^{\prime}} f^{+} d \mu \geqq 0$.

COROLlaRY 1. Given $\varepsilon>0$. With the hypotheses of Lemma 1 , there is a function $h_{\varepsilon} \in L^{1}$ and an integer $n_{0}=n_{0}(\varepsilon)$ such that
(i) $h_{s}^{-} \leqq f^{-}$,
(ii) $\int h_{\varepsilon} d \mu \leqq \int f d \mu$,
(iii) $\int_{B} h_{\varepsilon}^{-} d \mu<\varepsilon$ for all $B \in \mathscr{B}, B \subseteq A_{f}$,
(iv) $T_{m} f=T_{m} h_{\varepsilon}$ for all $m>n_{0}$. 
Proof. As $h_{n}^{-} \downarrow 0$ a.e. in $A_{f}$, choose $n_{0}$ such that $\int h_{n}^{-} d \mu<\varepsilon$ and let $h_{\varepsilon}=h_{n_{0}}$; (ii) follows from equation (2), since

$$
\int\left(f-h_{n}\right) d \mu=\sum_{0}^{n-1} \int\left(1-T_{i}\right) h_{i}^{+} d \mu \geqq 0
$$

and (iv) follows from equation (3).

3. The ratio limit theorem. Analogously to the proof of the Chacon-Ornstein theorem in [4], the following theorem now follows from Lemma 1.

THEOREM 1. If $\left\{T_{n}\right\}_{n \geqq 0}$ is a decreasing sequence of positive contractions in $L^{1}$, then, for all $f \in L^{1}, p \in L_{+}^{1}, \lim _{n \rightarrow \infty}\left[\left(T_{n} f\right)(\omega) /\left(T_{n} p\right)(\omega)\right]$ exists and is finite a.e. $(\mu)$ in

$$
B_{p}=\left\{\omega: \liminf _{n \rightarrow \infty}\left(T_{n} p\right)(\omega)>0\right\}
$$

Proof. Note that we may assume $f \geqq 0$.

We first apply Lemma 1 to $(f-a p)$ for $a>0$ to find $\int_{E}(f-a p) d \mu+\int_{E^{\prime}}(f-a p)^{+} d \mu \geqq 0$, where

$$
E=B_{p} \cap\{\omega: p(\omega)>0\} \cap\left\{\omega: \limsup _{n \rightarrow \infty} \frac{\left(T_{n} f\right)(\omega)}{\left(T_{n} p\right)(\omega)}=+\infty\right\} .
$$

Hence $\int(f-a p)^{+} d \mu \geqq \int_{E}(f-a p)^{-} d \mu$. Letting $a \rightarrow \infty$, we obtain $\mu(E)=0$. So

$$
\limsup _{n \rightarrow \infty}\left[\left(T_{n} f\right)(\omega) /\left(T_{n} p\right)(\omega)\right]<\infty .
$$

To show that, for any pair $a, b \in \mathscr{R}, a<b$, the set

$$
A=B_{p} \cap\{\omega: p(\omega)>0\} \cap\left\{\omega: \lim _{n \rightarrow \infty} \frac{\left(T_{n} f\right)(\omega)}{\left(T_{n} p\right)(\omega)}<a<b<\varlimsup_{n \rightarrow \infty} \frac{\left(T_{n} f\right)(\omega)}{\left(T_{n} p\right)(\omega)}\right\}
$$

is $\mu$-null, we apply the inductive procedure of [4, Lemma 5], using the function $h$ whose existence is guaranteed by Corollary 1 . To see that $T_{n} h / T_{n} p$ has the same limit behaviour as $T_{n} f / T_{n} p$, we note that $T_{n}(h-b p)=T_{n}(f-b p)$ for all $n>n_{0}(\varepsilon)$. Similarly, we find $f^{\prime}$ as in [4] and again $T_{n} f^{\prime}=T_{n} h=T_{n} f$ for sufficiently large $n$. Now $\mu(A)=0$ follows exactly as in [4]. This procedure, when applied to all pairs of rationals $a, b$ with $a<b$ gives convergence a.e. $(\mu)$ to a finite limit on the set $B_{p} \cap\{\omega: p(\omega)>0\}$. But, for any $n \geqq 0$, let $f_{1}=T_{n} f$, $p_{1}=T_{n} p ;$ then $T_{m} f_{1} / T_{m} p_{1}=T_{m} f / T_{m} p$ whenever $m>n$ and $T_{m} p>0$. So $\lim \left(T_{m} f_{1} / T_{m} p_{1}\right)=$ $\lim _{m \rightarrow \infty}\left(T_{m} f / T_{m} p\right)$ exists and is finite a.e. $(\mu)$ on the set $B_{p} \cap\left\{\omega:\left(T_{n} p(\omega)>0\right\}\right.$. As $T_{m} p>0$ infinitely often on $B_{p}$, we have proved the convergence on all of $B_{p}$.

4. Contractive projections. We proceed to identify the set $B_{p}$ in the case when $\left\{T_{n}\right\}_{n \geqq 0}$ is a decreasing sequence of pairwise commuting contractive projections. The set of all contractive projections on $L^{1}$ is partially ordered by the relation $\leqq$ defined by $P \leqq Q$ if and only if $P Q=P=Q P$ and a decreasing sequence of projections is decreasing in this ordering. 
Define the support of a measurable function $f$ by $S(f)=\{\omega: f(\omega) \neq 0\}$. Then we have:

LemMa 2. Let $f, g \in L_{+}^{1}$ and let $T$ be a positive linear operator on $L^{1}$. Then $S(f) \subseteq$ $S(g) \Rightarrow S(T f) \subseteq S(T g)$.

Proof. Put $f_{n}=f \wedge n g$. Then $T f_{n} \leqq n T g$, as $T$ is positive and linear. Hence $S\left(T f_{n}\right) \subseteq$ $S(T g)$. By [8, Lemma 3.9], $S(f) \subseteq S(g)$ if and only if $f_{n} \uparrow f$. Hence $T f_{n} \uparrow T f$ and so $S(T f)=\bigcup_{n} S\left(T f_{n}\right) \subseteq S(T g)$.

To apply Lemma 2 to a decreasing sequence $\left\{P_{n}\right\}$ of contractive projections, let $h \in L^{1}$, $h>0$ a.e. $(\mu)$. By Lemma 2 , if $g \in R\left(P_{n}\right)$, then $S(g) \subseteq S\left(P_{n} h\right)$. But $P_{n+1} h=P_{n} P_{n+1} h \in R\left(P_{n}\right)$; so $S\left(P_{n+1} h\right) \subseteq S\left(P_{n} h\right)$ and so $\left\{S\left(P_{n} h\right)\right\}$ is a decreasing sequence of sets. As $P_{n} \geqq 0$, $S\left(P_{n} h\right)=\left\{\omega:\left(P_{n} h\right)(\omega)>0\right\}$ and so Theorem 1 becomes

THEOREM 2. If $\left\{P_{n}\right\}$ is a decreasing sequence of positive contractive projections on $L^{1}$ and if $f \in L^{\prime}, h \in L^{\prime}$ and $h>0$ a.e. $(\mu)$, then $\lim _{n \rightarrow \infty}\left[\left(P_{n} f\right)(\omega) /\left(P_{n} h\right)(\omega)\right]$ exists and is finite a.e. $(\mu)$ in $\bigcap_{n=1}^{\infty} S\left(P_{n} h\right)$.

5. Application: Almost everywhere convergence for projections. In this section we follow the lead of Ito [6] and use the ratio theorem to prove the following theorem.

THEOREM 3. Let $(\Omega, \mathscr{B}, \mu)$ be a probability space and let $\left\{P_{n}\right\}$ be a decreasing sequence of pairwise commuting positive contractive projections on $L^{1}(\Omega, \mathscr{B}, \mu)$. If the sequence $\left\{P_{n} 1\right\}$, where 1 denotes the function taking constant value $1 \mu$-a.e., is uniformly integrable, then the sequence $\left\{P_{n} f\right\}$ converges $\mu-$ a.e. for any $f \in L^{1}$.

We need two lemmas. The first asserts the existence of a positive contraction $P$ on $L^{1}$, commuting with the $P_{n}$ and which is their limit in the strong operator topology. The statement and proof are close to that in [4], but we give the proof for the sake of completeness.

LEMMA 3. There is a positive contraction $P$ on $L^{1}$ such that $P_{n} P=P P_{n}$ for all $n$, and $P_{n} \rightarrow P$ in the strong operator topology.

Proof. As $\left\{P_{n} 1\right\}$ is a uniformly integrable sequence, the set $\left\{P_{n} 1\right\}$ is weakly sequentially compact, hence conditionally weakly compact, by the Eberlein-Šmulian theorem [3, p. 430]. As in [6, Lemmas 1 and 2], for any $B \in \mathscr{B}$, the set $\left\{P_{n} \chi_{B}\right\}$ is conditionally weakly compact, since $0 \leqq \chi_{B} \leqq 1$ implies $0 \leqq P_{n} \chi_{B} \leqq P_{n} 1$ for all $n$. So the set of all $f \in L^{1}$ such that $\left\{P_{n} f\right\}$ is conditionally weakly compact is total in $L^{1}$. But this implies that it is all of $L^{1}$, since a set of this kind is a closed linear manifold [3, Chapter II, 3.30].

Fix $f \in L^{1}$. Let $O(f)$ be the (strongly) closed convex hull of $\left\{P_{n} f\right\} . \quad O(f)$ is weakly compact, and is mapped into itself by $\left\{P_{n}\right\}$; hence we may apply the Markov-Kakutani fixed point theorem [3, Chapter $\mathrm{V}, 10.6]$ to deduce the existence of a fixed point $g \in L^{1}$. Now for given $\varepsilon>0$ there is a convex combination $\sum_{i=1}^{k} \lambda_{i} P_{n_{i}} f$ such that $\left\|g-\sum_{i=1}^{k} \lambda_{i} P_{n i} f\right\|<\varepsilon$. Choose 


$$
\begin{aligned}
& m>\max _{i=1 \ldots ., k} n_{i} ; \text { then } g=P_{m} g \text { and } P_{m}\left(\sum_{i=1}^{k} \lambda_{i} P_{n_{i}} f\right)=P_{m} f \text {. So } \\
& \qquad\left\|g-P_{m} f\right\|=\left\|P_{m}\left(g-\sum_{i=1}^{k} \lambda_{i} P_{n_{i}} f\right)\right\| \leqq\left\|g-\sum_{i=1}^{k} \lambda_{i} P_{n_{i}} f\right\|<\varepsilon .
\end{aligned}
$$

Therefore $P_{m} f \rightarrow g$ strongly. As the limit is unique, we may define $P: L^{1} \rightarrow L^{1}$ by $P f=g$ for each $f \in L^{1}$, with $f$ and $g$ related as above. The other statements follow at once.

The following results can be found in [11]. Let $Q$ be a positive contractive projection on $L^{1}$ and let $\Sigma=\{K \subset \Omega: K=S(f), f \geqq 0, f \in \mathscr{R}(Q)\}$. Then

(i) $\Sigma$ is a $\sigma$-ring;

(ii) if $f \in L^{1}$ has support contained in a member of $\Sigma$, then, for all $K \in \Sigma_{0}, Q\left(\chi_{K} f\right)=\chi_{K} Q f$;

(iii) each $f \in \mathscr{R}(Q)$ is $\Sigma$-measurable.

Now let $\Sigma_{n}=\left\{K \subseteq \Omega: K=S(f), f \geqq 0, f \in \mathscr{R}\left(P_{n}\right)\right\}$ and define $h_{n}=P_{n} 1, h=P 1, A_{n}=$ $\Omega \mid S\left(h_{n}\right)$ and $A=\Omega \mid S(h)$. Then Lemma 2 implies that $\left\{A_{n}\right\}$ is an increasing sequence of sets, and $\bigcup_{n} A_{n} \subseteq A$.

Lemma 4. $A=\bigcup_{n} A_{n}$.

Proof. We need to show that $A \subseteq \bigcup_{n} A_{n}$. or, equivalently, $\bigcap_{n} A_{n}^{\prime} \subseteq A^{\prime}$. Since $h_{n}$ is $\Sigma_{n}$ measurable, there is an increasing sequence $\left\{g_{m, n}\right\}_{m}$ of $\Sigma_{n}$-simple functions converging to $h_{n}$. Hence $P_{n}\left(g_{m, n} \chi_{A_{n}{ }^{\prime}}\right)=g_{m, n} P_{n} \chi_{A_{n^{\prime}}}$ and, by continuity, $P_{n}\left(h_{n} \chi_{A_{n}{ }^{\prime}}\right)=h_{n} P_{n} \chi_{A_{n}{ }^{\prime}}$. But $P_{n} h_{n}=$ $P_{n}^{2} 1=P_{n} 1=h_{n}$; so $h_{n}=P_{n} h_{n}=P_{n}\left(h_{n} \chi_{A_{n^{\prime}}}\right)=h_{n} P_{n} \chi_{A_{n^{\prime}}}$. Hence, on $A_{n}^{\prime}$, $P_{n} \chi_{A_{n^{\prime}}}=1$ a.e. $(\mu)$. But, on $A_{n}, 0 \leqq P_{n} \chi_{A_{n^{\prime}}} \leqq P_{n} 1=h_{n}=0$. Hence $0 \leqq P_{n} \chi_{A_{n^{\prime}}}=\chi_{A_{n^{\prime}}} \leqq h_{n}$ a.e. $(\mu)$. Therefore $h=\lim _{n} h_{n} \geqq \chi_{\Gamma_{n} A_{n^{\prime}}} \geqq 0$, since the $A_{n}^{\prime}$ form a decreasing sequence. Hence, on $A, \chi_{n_{n} A_{n^{\prime}}}=0$ a.e. $(\mu)$, or $\chi_{n} A_{n^{\prime}} \leqq \chi_{A^{\prime}}$. This means that $\bigcap_{n} A_{n}^{\prime} \subseteq A^{\prime}$, i.e., that $A \subseteq \bigcup_{n} A_{n}$.

Proof of Theorem 3. We may assume that $f \geqq 0$. By the ratio limit theorem, $\lim \left(P_{n} f / P_{n} h\right)$ exists a.e. $(\mu)$ on $A^{\prime}$. But $h$ is invariant under all the $P_{n}$; so the $\operatorname{limit}$ is $(1 / h) \lim P_{n} f$ on $A^{\prime}$. Hence $\lim P_{n} f$ exists a.e. $(\mu)$ on $A^{\prime}$. On $A, \lim \left(P_{n} f\right)(\omega)=0$ a.e. $(\mu)$; we know that $\left\{\omega:\left(P_{n} f\right)(\omega)=0\right\} \supseteq A_{n}$, by Lemma 2. The $\left\{A_{n}\right\}$ are increasing to $A ;$ so $\lim \left(P_{n} f\right)(\omega)=0$ a.e. ( $\mu$ ) on $A$.

\section{REMARKS.}

1. For functions with support contained in $S(h), P$ acts as conditional expectation operator with respect to $\Sigma$.

2. The assumption of commutativity is essential in Theorem 3, though the ratio theorem holds without it. In fact, Lemma 3 does not generalise. Consider $\Omega=[0,1], \mathscr{B}=$ the Borel 
field, $\mu=$ Lebesgue measure. Let $E_{n}$ be the set of all $\omega \in \Omega$ with binary expansion $\omega=$ .$x_{1} x_{2} \ldots x_{n} \ldots$ such that $x_{n}=0$. Then let $P_{n} f=2\left(\int_{0}^{1} f d \mu\right) x_{E_{n}} . \quad\left\{P_{n} f\right\}$ clearly does not converge strongly for any $f \in L^{1}$, but, since $\left\{P_{n} 1\right\}$ converges weakly to 1 , it is uniformly integrable. This example is due to Dona Strauss (oral communication).

\section{REFERENCES}

1. E. Bishop, Foundations of a constructive analysis (New York, 1967).

2. R. G. Douglas, Contractive projections on an $L^{1}$-space, Pacific J. Math. 15 (1965), 443-462.

3. N. Dunford and J. T. Schwartz, Linear operators, Part I (New York, 1964).

4. E. Hopf, On the ergodic theorem for positive linear operators, J. Reine Angew. Math. 205 (1961), 101-106. $107-124$

5. A. and C. Ionescu Tulcea, Abstract ergodic theorems, Trans. Amer. Math. Soc. 107 (1963),

6. Y. Ito, Uniform integrability and the pointwise ergodic theorem, Proc. Amer. Math. Soc. 16 (1965), 222-227.

7. M. Jerison, Martingale formulation of ergodic theorems, Proc. Amer. Math. Soc. 10 (1959), $531-539$.

8. S. Kakutani, Concrete representations of abstract $(L)$-spaces and the mean ergodic theorem, Ann. of Math. 42 (1941), 532-537.

9. M. M. Rao, Generalised martingales, Contributions to probability and ergodic theory, Springer, Lecture Notes in Mathematics 160 (Berlin, 1970).

10. G. C. Rota, Une théorie unifiée des martingales et des moyennes ergodiques, C.R. Acad. Sci. Ser A 252 (1961), 2064-2066.

11. D. E. Wulbert, Characterisation of conditional expectation operators, Pacific J. Math. 34 (1970), 285-288.

\section{UNIVERSITY OF HULL}

